\title{
The Weak Hydrogen Bond: Applications to Structural Chemistry and Biology(International Union of Crystallography Monographs on Crystallography, 9). By Gautam R. Desiraju (grdch@uohyd.ernet.in, University of Hyderabad) and Thomas Steiner (steiner@chemie.fu-berlin.de, Freie Universität Berlin). Oxford University Press: Oxford, UK. 1999. 507pp. \$ 150. ISBN 0-19-850252-4.
}

\author{
Shu-Kun Lin \\ Molecular Diversity Preservation International (MDPI), Saengergasse 25, CH-4054 Basel, Switzerland \\ Tel: +41 79322 3379, Fax: +41 61302 8918, E-mail: lin@mdpi.org
}

Received: 7 September 1999 / Published: 10 October 1999

This book is an important monograph for those working on the related structural chemistry of the weak hydrogen bond. It has about 1000 citations. A large number of stick and ball structures are given in the book, depicting the hydrogen bonds and their environments in crystals.

Other books, particularly several recently published ones on the hydrogen bond are recommended on page 4. Chapter 1 gives a brief and very nice introduction to the historical background and definition of the hydrogen bond in general and the weak hydrogen bond in particular. Personally I am fascinated by the hydrogen bond topic because of my great interest in entropy concepts. Pauling's paper on residual entropy of ice [1] is famous as it used the name hydrogen bond for its first time, as also pointed out in this book. In this paper [1] Pauling elegantly applied his resonance concept to the structure of ice.

In German speaking countries, chemists normally use "H-Brücke" (H bridge) instead of hydrogen bond. The authors commented that "the use of the word bridge is hardly objectionable."

Normally weak hydrogen bonds are of $-\mathrm{C}-\mathrm{H} \cdots \mathrm{X}$ type, where the $\mathrm{H}$ donor is from a $\mathrm{CH}$ of $-\mathrm{C}-\mathrm{H}$, while the normal, typical hydrogen bond has $-\mathrm{O}-\mathrm{H}$ and $-\mathrm{N}-\mathrm{H}$, etc., as $\mathrm{H}$ donors. If the acceptor is a structural moiety with several centers, the hydrogen bond is called non-conventional. In some cases,

(C) 1999 by the authors. Reproduction of this article, by any means, is permitted for noncommercial purposes. 
the hydrogen bond is classified as weak because the $\mathrm{H}$ acceptor is very weak, even though the $\mathrm{H}$ donor is normal. Chapters 2 and 3 categorises different weak hydrogen bonds.

Chapter 4 is entitled "The weak hydrogen bond in supramolecular chemistry". Many crystal structures are used to illustrate the importance of the weak hydrogen bond.

The final chapter (Ch. 5) gives an account of the special significance of the weak hydrogen bond in biological molecular structures. Section 5.1.1 has a very interesting discussion regarding the dynamics in the static structure of biological structures. The spontaneous self-organization of biomolecules may be divided into two general weak effects. One is due to the interaction of atoms or moieties of the same or very similar properties (e.g., hydrophobic interaction and $\pi$-stacking). The other is the socalled complementarity where the interacting partners are of different properties (e.g., one is $\mathrm{H}$ donor, the other is $\mathrm{H}$ acceptor) [2]. The experimental facts presented in this monograph are very helpful in understanding these two kinds of effects [2], particularly the complementarity, i.e., whether they are weak or not.

\section{References and Notes}

1. (a) Pauling, L. The structure and entropy of ice and of other crystals with some randomness of atomic arrangement. J. Am. Chem. Soc. 1935, 57, 2680-2684. (b) Giaque, W. F.; Stout, J. W. The entropy of water and the third law of thermodynamics. The heat capacity of ice from 15 to $273 \mathrm{~K}$. J. Am. Chem. Soc. 1936, 58, 1144-1150. (c) Long, E. A.; Kemp, J. D. The entropy of deuterium oxide and the third law of thermodynamics. Heat capacity of deuterium oxide from 15 to $298 \mathrm{~K}$. The melting point and heat of fusion. J. Am. Chem. Soc. 1936, 58, 1829-1834.

2. Lin, S.-K. Similarity rule and complementarity rule, Chimia 1999, 53, 383.

(C) 1999 by the authors. Reproduction of this article, by any means, is permitted for noncommercial purposes. 